\title{
Building an Informal Ontology to Support Collaborative Network Operation: A Case Study
}

\author{
Carla Pereira ${ }^{1,2}$, Cristóvão Sousa ${ }^{1,2}$, and António Lucas Soares ${ }^{1,3}$ \\ ${ }^{1}$ INESC Porto, Campus da FEUP, Rua Dr. Roberto Frias, 378, 4200-465 Porto, Portugal \\ ${ }^{2}$ DEI, FEUP, University of Porto, Rua Dr. Roberto Frias, sn 4200-465 Porto, Portugal \\ ${ }^{3}$ ESTGF-IPP, Apartado 2054610 - 156 Felgueiras, Portugal \\ csp@inescporto.pt, cpsousa@inescporto.pt, asoares@inescporto.pt
}

\begin{abstract}
Looking at collaborative networks of organizations as socialtechnical systems, information and knowledge sharing implies sharing a set of common conceptual structures across organizations. Through this paper, we describe a case study on how to construct meaningful collective conceptualizations by means of a new approach based on conceptual blending theory.
\end{abstract}

Keywords: conceptualization, ontology development, meaning construction, collaborative networks.

\section{Introduction}

The early phases of setting up information and knowledge management architectures for inter-organizational teams (e.g., large scale international R\&D or systems engineering projects) are complex and problematic mainly due to the actor's heterogeneous professional and cultural backgrounds. The big challenge stands on the proper definition, in short-term, of the concepts and relations, which are the basis of the information and knowledge architecture to be common to the collaborative network. Like this, considering a common conceptualization of a given reality as the cornerstone for information and knowledge sharing among a network of social actors, the core problem is how to create suitable socio-technical tools to support the collaborative process of creating semantic artifacts to be operationalized in some short-term business opportunity.

Sharing information and knowledge implies sharing a set of conceptual structures between network partners. They need to negotiate a common vocabulary, meaning of concepts and their relations, to develop the information organization system. The problems raised by information and knowledge sharing in the context of collaborative networks have been researched in the last decade (and is still being researched) in the so-called Ontology Engineering community. After a deep analysis of the state-of-theart in this area one can conclude that the current underpinnings of ontology engineering are not enough for the future challenges of building semantic artifacts, i.e., the current underpinnings of ontology engineering do not give an appropriate answer to the new challenges raised by the every time larger need of collective construction of meaning. Even though the most used definition of ontology [1] "An ontology is a formal, explicit 
specification of a shared conceptualization", underlines the collaborative construction of conceptualizations in the scientific context, it is established that: "While different degrees of formalizations have been well investigated and are now found in various ontology-based technologies, the notion of a shared conceptualization is neither wellexplored, nor well-understood, nor well-supported by most ontology engineering tools" [2]. Our view is that ontology engineering needs a "socio-cognitive turn" in order to generate tools that are really effective in copying the complex, unstructured, and highly situational contexts that characterize a great deal of information and knowledge sharing in businesses collaboration. This line of research is therefore directed towards the application of cognitive semantic results in the creation of artifacts acting as sociotechnical devices supporting the view that meaning socially constructed through collaboration and negotiation. The first line of this research work deals with the application and extension of the Conceptual Blending Theory (CBT) [3] to the realm of collaborative semantic tools. The practical application of our approach is to support the co-construction of semantic artifacts by groups of social actors placed in organizational contexts interacting towards a set of common objectives. We propose a new method to support de collaborative construction of semantic artifacts with special focus in the initial phase of the process, the conceptualization phase (to obtain a detailed description of the CBT based method, see $[4,5])$.

In this paper, are described the usage of the method in the scope of a large European project, $\mathrm{AC} / \mathrm{DC}^{1}$, showing the main results and lessons learnt with this case study.

\section{Application Case: Building a Project Informal Ontology}

This experiment was carried out within a trans-national (European) project, AC+DC project, in the area of industrial (automotive) engineering. In this project, a consortium of major European car manufacturers, suppliers, and research institutes develop the "dynamic supply chain collaboration" concept that changes the conventional automotive terms of delivery to a highly reactive "5-Day-capable" system that radically cuts down inventories in the supply network. This is a huge and complex project, as it involves 19 partners, from 7 countries and 9 tasks grouped in 3 work packages. One of the work packages aims at building an ontology to be used in several tasks of the project. The general goal of the $\mathrm{AC}+\mathrm{DC}$ ontology is to facilitate a common and precise understanding of the concepts used by all partners in the several project activities. The resulting common vocabulary should match standardized terms of automotive production domain and the particular concepts on development in the project. The ontology development task started initially without a supporting methodology or even a clear vision of the ontology goals and scope. Without the concern of clarifying the general goal of the ontology, and without discussion of this with the partners, began its development. The first obstacle comes at this very early stage, where to start and how to start? The first version of the ontology was presented. Comprised data models which included concepts, relations, properties and data types. Basically, the first version was based on a software application data model related to the planning and

${ }^{1} \mathrm{http} / / /$ www.acdc-project.org/public/ 
production scheduling. The terms contained in the above example, were made available to other members in the form of a "Protégé Project". This version came out from expert domain partners with lack of knowledge in the development of ontologies. They were not aware about the real purpose of "Protégé", as well as its limitations and capabilities. Therefore, the result was not as it was intended initially. Moreover, when creating the ontology, the process itself was lame and neither truly collaborative nor guided by basic collaborative rules, essential to support the process. The Protégé files were exchanged by e-mail to all partners, which, by their turn, could reply some comments on the ontology. The participation was weak; probably due the lack of knowledge of the Protégé software and its associated notation, which constituted a barrier, making the process, move forward without the contributions of all.

Within this context, the authors took the opportunity to set-up an action-research project aiming at, from the one side, to help the project to develop its ontology and, on the other side, to create knowledge about the collaborative construction of ontologies by designing and undertaking a set of experiments. From the preliminary analysis of the problem, jointly with the project team, the following general requirements were derived: (1) the goals and scope of the ontology should be clearly stated, even if not completely detailed; this is of utmost importance to guide the conceptualization process; (2) there is a clear need for a method and tools to support the conceptualization process; only with such a support a collaborative process is feasible; (3) There is a need to use tools and techniques that can be used by all involved regardless of their training. Initially the emphasis should not be in formal aspects related to the ontology codification. The concern should focus on the informal knowledge representation that will constitute the ontology; and (4) the possibility of reusing other ontologies should be evaluated. For example, the analysis of models created in the project ILIPT ${ }^{2}$ shows that the results can be used to prepare the initial proposal for a shared conceptualization.

Hereupon, the authors attempted to see whether it would be possible to reuse existing ontologies in supply chain management domains. From the studied ontologies, [8] and [9] were based on SCOR model; others such as [13] are focused inside organizations but not among them; and The United Nations Standard Products and Services Code which provides an open, global multi-sector standard for efficient, accurate classification of products and services. These ontologies cover very specific subdomains making them difficult to reuse. There are also several upper-level ontologies which are too abstract to be applied in particular situations. Since collaboration concepts behind supply chains and their requirements could be fundamentally different, there is no standard ontology, which would be detailed enough to be applicable in every practical case.

In this experimental phase four tools were used to support the collaborative conceptualization process. For the joint construction of a conceptual representation, Concept Maps supported by CmapTools ${ }^{3}$ and Semantic Media Wiki ${ }^{4}(\mathrm{SMW})$ were used. CmapTools was used essentially to support the frame definition, preliminary proposal and input spaces creation $[4,5]$. The blend services and negotiation process was assured by SMW. An integration service between CmapTools and SMW was developed

\footnotetext{
${ }^{2}$ http://www.ilipt.org/public/

${ }^{3}$ http://CMAP.ihmc.us/conceptmap.html

${ }^{4}$ http://semantic-mediawiki.org/wiki/Semantic_MediaWiki
} 
allowing the discussion of input spaces and the annotation of concepts within each input space, gathering information to run the blend. To support the blend spaces creation, together with CmapTools features and SMW, the text mining tool, TermExtractor $^{5}$, capable to extract relevant terms in the interest domain, by submitting an archive of domain-related documents in any format was used. IATE was used, occasionally, to search for the correct correspondent terms chosen to name a concept, in several languages, according to a specific domain.

The application case scenario was identified and some preliminary studies made, leading to the following experiment context: Four teams from four different organizations (geographically dispersed) participated in the domain conceptualization. Two of those teams (Team1 and Team2), from two different organizations (Org1 and Org2), were domain experts from academic and professional areas respectively; another team (Team3) from another organization (Org3) was composed by experts in information and knowledge management and in collaborative networks; the fourth team (Team4) from another organization (Org4), beyond their academic expertise in the specific domain, has a reasonable understanding about ontologies. The experiment started to be more formally structure, firstly by establishing the roles of each actor: 1) Contributor: all team members should play this role contributing for the improvement and enlargement of the current version of the $\mathrm{AC}+\mathrm{DC}$ conceptualization. The contributor responsibilities are to make inputs to the shared conceptualization by proposing and discussing concepts and relationships; 2) Facilitator: responsible by facilitating the discussion/negotiation around the conceptualization. Teams 2, 3 and 4 were assigned the role of contributor; team 1 was assigned the role of facilitator. The first step, the context frame definition, was accomplished in the initial formal meeting and helped to define the context, goal and mission of the ontology development task, as well as the scope and boundaries of the conceptualization. It was then decided that initially the ontology would contribute to a common understanding of the concepts used and needed by all partners in the various project activities, their goals may be expanded in the next iterations of the process. Thus, considering the original purpose it was decided that the ontology should in the first instance, reorganize itself around the main concept in developing the project, "Dynamic Supply Loops" (DSL), this being his main focus. Along with the capture, organization and justification for each term inherent to DSL, it would be necessary to identify concepts, common in the automotive production field and supply chain management, to be able to explain and contextualize the new concepts. With the main focus in the dynamic supply chain collaboration concept, more precisely in the DSL concepts, the team created an initial shared conceptualization guided by the following focus question: "what processes, activities and information are involved in the DSL network planning model, allowing collaboration in entire supply chain in feedback loops?" The resulting conceptualization was presented in a concept map, defining the scope and boundaries of the conceptualization process, i.e., this result together with the goals initially defined for the ontology constituted the "context frame". Afterwards, the conceptualization of the several process and activities was initiated, which means detailing the DSL. The first process to be treated was the "Production Planning Process". After the construction of the first concept map (the preliminary proposal, see figure 1), an object was created in SMW in

\footnotetext{
${ }^{5} \mathrm{http}: / / \mathrm{lcl} 2 . u n i r o m a 1 . i t / T e r m E x t r a c t o r$
} 
order to aggregate ideas and propositions about the Production Planning Process. Thus, all the teams in the project were able to extend the first shared domain conceptualization, contributing with their own inputs (changes that were made in the input spaces (on CmapTools or SMW) and which were published. All members could see the other member's inputs. The creation of new inputs by each team's domain expertise come from the results from TermExtractor tool document analysis, other documents produced by the several project work packages, the logistics area of SAP dictionary ${ }^{6}$, the terms and glossary about supply chain management proposed by [10] and, most important of all, by the conceptual structures identification in the experts mind when they interact with the other member's proposals.

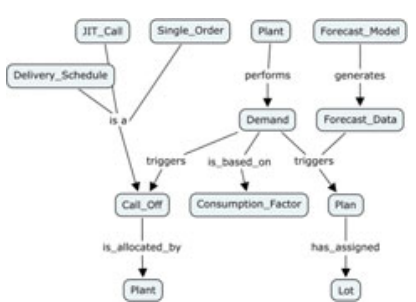

Preliminary proposal (Production Planning Process)

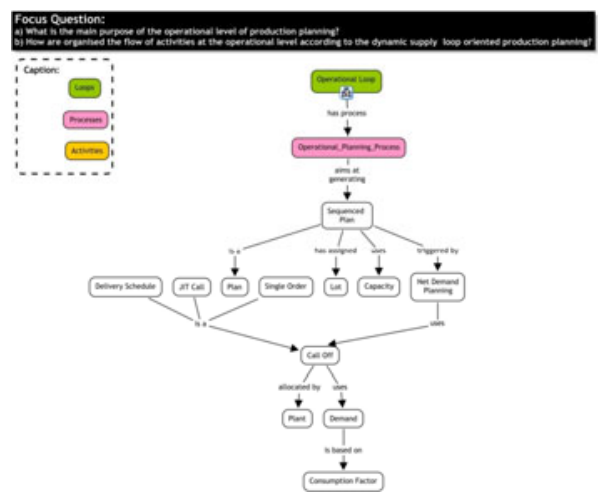

Production planning (operational loop) - shared conceptualization

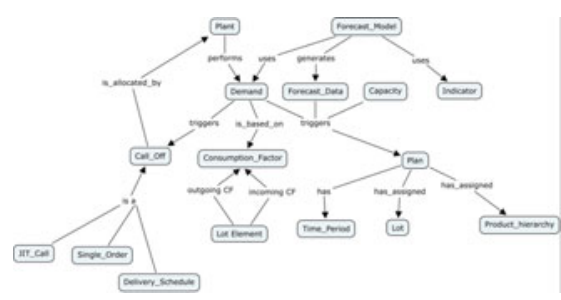

Partner conceptualization proposal (example of an input space)

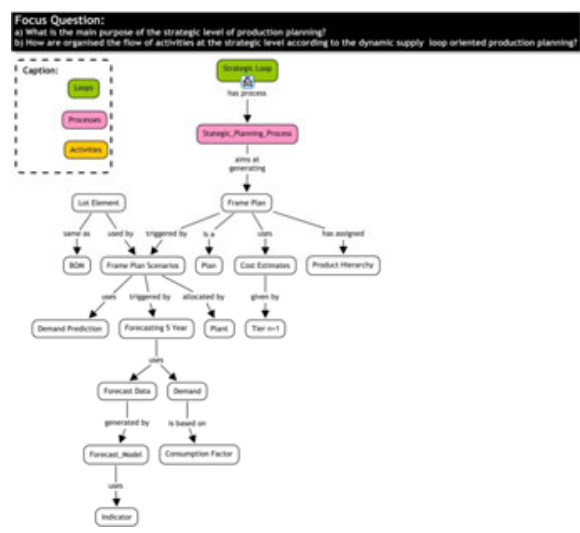

Production planning (strategic loop) - shared conceptualization

Fig. 1. Excerpt of the concept maps created during the collaborative conceptualization process

The discussion is initiated using the functionalities of SMW. Following the end of a discussion, the approved (consensual) concepts, can be imported directly to the CmapTools Client instance of each organization, updating the personal map in focus. An excerpt of the final shared conceptualization achieved is presented in figure 1.

\footnotetext{
${ }^{6}$ http://help.sap.com/saphelp_46c/helpdata/En/35/2cd77bd7705394e10000009b387c12/ frameset.htm
} 
Technically, all this process is supported by the CmapTools and Semantic Media Wiki. CmapTools aggregates in the server all the input spaces, therefore users are able to publish them in SMW. With TermExtractor the enrichment of the current conceptualization by the discovery of new terms and the supported validation of the existing ones was achieved. All the input spaces have the same conceptual structure and the person in charge for coordinating the process performs the upload of the final (consensual) map into the server. The concept maps present in the CmapServer, comprise the generic space.

\subsection{Main Observations and Recommendations}

The trend towards the development of formal models early in the process, it is confirmed to be a bad principle, leading to poor results and demanding an excessive concern by the participants with the formal semantics restrictions (e.g., starting development using tools such as Protégé). At the beginning, becomes essential for participants to be focused on the task of building the conceptual model in an environment without constraints, trying to express they knowledge about the domain. The lack of prior analysis and knowledge of methods and tools to support the collaborative construction of a conceptualization, result in an inadequate selection of them. For example, the type of players in the process, knowledge areas and technologies and techniques proposed, are factors that may be decisive for the success of the overall process. There was a high receptiveness regarding the use of concept maps as visual representation technique. This experience showed that conceptual maps are a suitable tool to be used during the collaborative conceptualization process, because in this phase "completeness is more important neatness and rigor" [11]. From our observation and from the interviews, we concluded that discussing the problem using a domain-specific vocabulary supported by a visually oriented, easy to use, informal tool, effective results could be achieved in a relatively short time. Our goal is to allow that the users could start informally, the construction of a (non-computational) "knowledge base" without having to commit to a particular knowledge representation, and without having to translate their know-how into any particular knowledge representation format. After the informal knowledge is built up, its structure may become more obvious. Thus, users could then begin to gradually coerce the concept maps to conform to the formal semantic system.

In the following paragraph we share some lessons learnt with this experiment. (1) Initial social and cultural analysis: the social and cultural analysis of the various partners, although brief, is necessary to design the process. For example, the decision by a process with rules and procedures more or less rigid may depend on the type and number of players in the process; (2) Appropriate definition of the context frame and road map: The starting point of this case study shows clearly the importance of these tasks. After the initial definition of the context frame and road map, is equally important that in the previous specified time periods, go backwards and review the following questions: What do we have? What do we want? and How to get there? These questions allow the team to evaluate the forces and weaknesses of current situation; (3) Rules to organize the process and motivate the participation: The evaluation showed that the majority of users were passive in their participation. Automatic notifications of all teams whenever changes exist, version control and definition of a time 
frame in which the proposals can be discussed are fundamental to better organize the process, and motivate the participants. Therefore, if no one present suggestions during the time period defined, it means that agreement exists. Every time there's a change in any discussion item within the process, users should be informed and invited to comment the new proposals. All these procedures must be agreed initially. Should be defined, also initially, a strategy that will lead the negotiation process. The use of workflow mechanisms allow us to manage the process and maintain the control of the various conceptualization proposals that are created. (4) Always try to reduce the complexity of the process: The collaborative process of conceptualization is really complex because of the high number of areas, processes and activities, among others. One way to deal with this is to follow some rules such as [12]; (5) Project generated documentation as an enabler: The continuous production of project documentation is a way to validate and improve the conceptualization. On the other hand, the consensual conceptual structure, agreed so far, should be used in the production of new deliverables in order to standardize the contents of each deliverable. By this reason it's easy to share and understand the meaning of the concepts in the domain; (6) First version of the conceptualization: the necessity of the preliminary proposal was identified in the first experiment. Even with only the high-level concepts, this first version proved to be, a good way to focus the discussion and guide the process; (7) The existence of a negotiation space (blend space): The existence of a blend space provided more reliability, collaboration and agility to the process of conceptualization. This was due to the fact that the inputs for blend were based on project produced documentation, as well as other important resources selected by the domain experts. This resulted in a high level of acceptance of the proposals. (8) Carefully selection of the information resources used as inputs in the blend space: The results obtained in the blend depend directly on the information sources used. The blend results can be accepted with more or less support, according to the provided inputs during its creation.

The work performed under the project AC/DC triggered the attention of the remaining project partners, especially the leader, Continental Teves AG for the process and its benefits in creating semantic artifacts. Continental is a growing organization and recently acquired other companies. One of the biggest challenges that Continental has faced has been the implementation of their processes and knowledge sharing associated with the operationalization of them. Continental faced this process based on concept maps as a simple way to provide information about their processes and for sharing how they are to be implemented. The technological framework (SemSys4CCM platform, presented in [4]) inherent to the experience carried out under this project, also served as a inspiration for Continental to start its new approach to knowledge sharing, which reveals the satisfaction of the project leader with the procedures used during the informal ontology building.

\section{Conclusions and Further Work}

It is important to refer that the correct understanding of this paper implies the reading of $[4,5]$, the limitation on the number of pages required to leave the background some important issues, but already published and can be found in the mentioned papers. The approach advocated in this paper proposes a shift in the process of creation of 
semantic artifacts from a "semantic artifact engineering" perspective to an "actorartifact co-evolution" one. Socio-semantics is the scientific umbrella to this approach, which is also inspired in cognitive semantics and networking social theories (see [6, 7]). The case studies accomplished allowed us to design the architecture of a collaborative environment architecture that will support all steps of the method (see [4]). Future work involves: (1) considering the conceptualization as a social process, to explore the socio-semantic framework aiming at specifying mechanisms to support the social activity involved in the shared meaning creation; (2) Apply social network analysis to study the influence of social relationships within the meaning negotiation process; (3) conclusion of the SemSys4CCM platform development; (4) Enrichment of the input spaces and blend space using knowledge of terminology and computational linguistic areas; and (5) extend the CBT based method to support the development of multilingual conceptualizations.

\section{References}

1. Gruber, T.R.: A Translation Approach to Portable Ontology Specifications. Knowledge Acquisition 5(2), 199-221 (1993)

2. Staab, S.: On understanding the collaborative construction of conceptualisations. In: International and Interdisciplinary Conference "Processing Text-Technological Resources" at the Center for Interdisciplinary Research, Bielefeld University, March 13-15 (2008)

3. Fauconnier, G., Turner, M.: Conceptual Integration Networks. Published in Cognitive Science 22(2), 133-187 (1998)

4. Pereira, C., Sousa, C., Soares, A.: A socio-semantic approach to collaborative domain conceptualization. In: Meersman, R., Herrero, P., Dillon, T. (eds.) OTM 2009 Workshops. LNCS, vol. 5872, pp. 524-533. Springer, Heidelberg (2009)

5. Pereira, C., Sousa, C., Soares, A.: Short-Term Semantic Consensus: Towards Agile Ontology Specification for Collaborative Networks. In: Leveraging Knowledge for Innovation in Collaborative Networks. IFIP Advances in Inf. and Com. Technology. Springer, Boston (2009)

6. Evans, V., Green, M.: Cognitive Linguistics: an introduction. Edinburgh University Press, Edinburgh (2006)

7. Cahier, J.-P., Zaher, L.H., Leboeuf, J.-P., Guittard, C.: Experimentation of a socially constructed "Topic Map" by the OSS community. In: Proc. of the IJCAI 2005 workshop on KMOM, Edimbourg (2005)

8. Ureten, S., Ilter, H.K.: Supply Chain Management Ontology: Towards an Ontology-Based SCM Model. In: Proc. of the Fourth Int. Logistics and Supply Chain Management Congress (2006)

9. Fayez, M., Rabelo, L., Mollaghasemi, M.: Ontologies for Supply Chain Simulation Modeling. In: Proc. of the 2005 Winter Simulation Conference (2005)

10. Vitasek, K.: Supply Chain Management, Terms and Glossary (2008)

11. Kremer, R.: Concept Mapping: Informal to Formal. Appeared in Proceedings of the International Conference on Conceptual Structures, University of Maryland (1994)

12. Gómez-Gauchía, H., et al.: Two-layered approach to knowledge representation using conceptual maps and description logics. In: Concept Maps: Theory, Methodology, Technology, Proc. of the First Int. Conf. on Concept Mapping (2004)

13. Maier, A., Schnurr, H.-P., Sure, Y.: Ontology-Based Information Integration in the Automotive Industry. In: Fensel, D., Sycara, K., Mylopoulos, J. (eds.) ISWC 2003. LNCS, vol. 2870, pp. 897-912. Springer, Heidelberg (2003) 